\title{
The performance of robust control chart for change in variance.
}

\begin{abstract}
A control chart for detecting shifts in the variance of a process is developed for the case where the nominal value of variance is unknown. The Shewhart S control chart is one of the most extensively used statistical process control techniques developed for control process variability based on essential assumption that the underlying distribution of the quality characteristic is normal. However, this chart is very sensitive to the occurrence of occasional outliers. As an alternative, robust control charts are put forward when the underlying normality assumption is not met. In this study, a robust control chart for the process standard deviation $\sigma$ by means of a robust scale estimator is proposed. The presented robust method seems to yield a better performance than the Shewhart method and had good properties for the contaminated and heavy-tailed distribution for moderate sample sizes. The proposed robust Modified biweight A chart acts as an alternative for practitioners who are interested in the detection of permanent shifts in the process variance whereby the presence of occasional outliers is usually associated with the occurrence of common causes.
\end{abstract}

Keyword: Change point; Statistical process control; Out-of-control; In-control; outlier; Robust; Modified biweight a scale estimator. 